\title{
Integrated Quantum Well Bipolar Devices for Tunable Detection and Optical Logic Applications
}

\author{
P. Bhattacharya, J. Singh, S. Goswami and W-Q. Li \\ Solid State Electronics Laboratory \\ Department of Electrical Engineering and Computer Science \\ The University of Michigan, Ann Arbor, MI 48109-2122, USA
}

\begin{abstract}
The properties of exciton-based quantum well devices suitable for low-power photonic switching and logic applications and wavelength selective detection will be described. The basic device is a heterojunction bipolar transistor with MQW collector region monolithically integrated with a $\mathrm{p}-\mathrm{i}(\mathrm{MQW})-\mathrm{n}$ modulator, realized by single-step epitaxy. The integrated controller-modulator device, by virtue of its unique photocurrent-voltage characteristics, exhibits integrating-thresholding properties. These properties have been exploited to demonstrate cascadability, fan-out, optoelectronic amplification, special logic functions, programmable optical/electronic memory capabilities, and wavelength selective detection with GaAs/AlGaAs heterostructure devices.
\end{abstract}

\section{INTRODUCTION}

Excitonic transitions arise from electron-hole states coupled via the Coulombic interaction. In presence of an electric field, the excitons dissociate at small electronic fields $(\leq 10 \mathrm{kV} / \mathrm{cm})$ in bulk semiconductors, but in quantum wells where the exciton is confined by strong barriers, the exciton position shifts to lower energies with applied bias without significant loss in the absorption peak. This effect known as QCSE [1-4] can be exploited to produce an assortment of important characteristics illustrated schematically in figure 1. Figure $1(\mathrm{a})$ shows the commonly used $\mathrm{p}-\mathrm{i}(\mathrm{MQW})-\mathrm{n}$ structure for applying the reverse bias for QCSE. The absorption coefficient displays the features shown in figure $1(\mathrm{~b})$ where the two peaks correspond to the heavy hole $(\mathrm{HH})$ and light hole $(\mathrm{LH})$ transitions. When an electric field is applied, the spectra moves towards lower energy and this feature cannot only be exploited for optical 


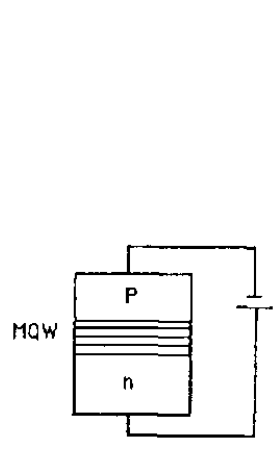

(a)

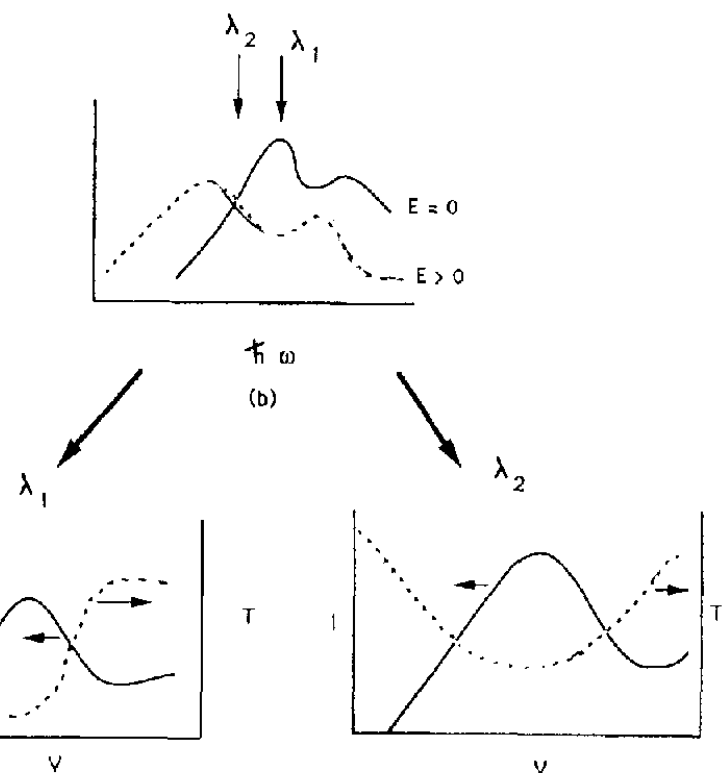

(c)

Figure 1 (a) A p-i(MQW)-n modulator; (b) excitonic absorption spectra in absence and presence of a field; (c) current-voltage and transmittance-voltage curves at different wavelengths.

modulation, but also has a very interesting effect on the photocurrent. At room temperature, the photocurrent is essentially proportional to the photons generated, even at fairly low applied bias. The photocurrent versus applied bias curves can thus be tailored quite efficiently as shown in figure 1 (c) where we show the photocurrent and transmittance through the structure at two different wavelengths $\lambda_{1}$ and $\lambda_{2}$ whose position is indicated in figure $1(b)$. As can be clearly seen very different forms of I-V curves result. The photocurrent-voltage characteristics just described can be exploited for wavelength selective detection and for the design of novel optical logic and switching devices and even an optically controlled oscillator. In what follows, these concepts and their realization are described.

\section{WAVELENGTH SELECTIVE DETECTION}

An important feature of the excitonic transitions is the sharpness of the resonances. The I-V curves are thus very sensitive to the input wavelength as discussed before. If the input signal, say in a communication channel, is coming digitially and serially in one of the several wavelengths, the excitonic detector can be tuned to either receive it or discard it. This ability is, of course quite useful, but is not as useful as the ability to do so even if several wavelength signals were coming in parallel. A simple $\mathrm{p}-\mathrm{i}(\mathrm{MQW})-\mathrm{n}$ diode does not have such a discrimination, but the simple circuit shown in figure 2(a) which carries out a differentiation of the photocurrent has a strong 


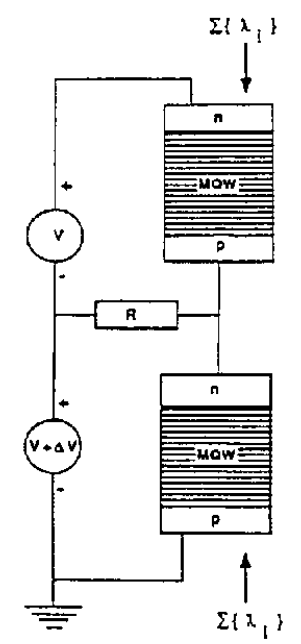

(a)

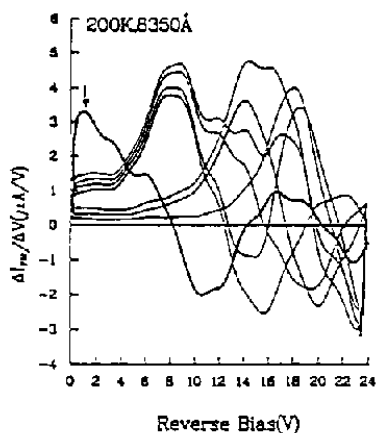

(b)

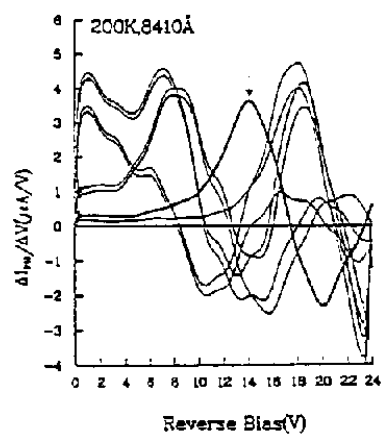

(d)

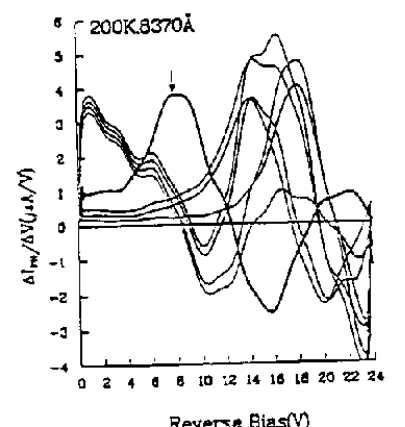

(c)

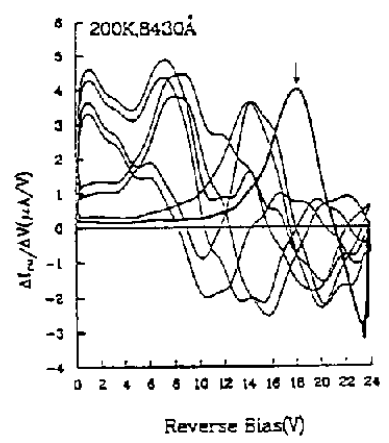

(e)

Figure 2 Schematic for the photocurrent differentiation studies circuits along with some typical results for wavelengths selective applications.

wavelength selectivity even when a number of wavelengths are impinging in parallel. In figure $2(\mathrm{~b})-2(\mathrm{e})$ we show the values of $\Delta I_{\mathrm{ph}} / \Delta V$ at $200 \mathrm{~K}$ for different states of a 4-channel parallel input. A GaAs/AlGaAs MQW structure has been used. The four wavelengths used are $8350 \AA, 8370 \AA, 8410 \AA$ and $8930 \AA$. The results in bold are for the case when the ON state of only the channel b) $\lambda_{i}=8350 \AA$; c) $\lambda_{i}=8370 \AA$; d) 8410 and e) $\lambda_{i} 8430 \AA$. Each of these channels can be distinguished regardless of the input of other channels if the device is biased at the points marked by the arrows in the figures. At these bias points if $\Delta I_{p h} / \Delta V$ is greater than $2 \mu \mathrm{A} / \mathrm{V}$, the particular $\lambda_{i}$ bit is ON (regardless of input in the other channels) and if it is less than $2 \mu \mathrm{A} / \mathrm{V}$, it is OFF.

It has been shown that up to six channels can be selectively detected [5]. The number of channels can be further increased if the MQW structures are not made up of same size single quantum wells, but are formed from say two different size wells so as to remove the dip in absorption between the HH and LH transitions. The step function absorption profile used in the differentiation mode (figure 2(a)) is expected to have a very high wavelength selectivity. 


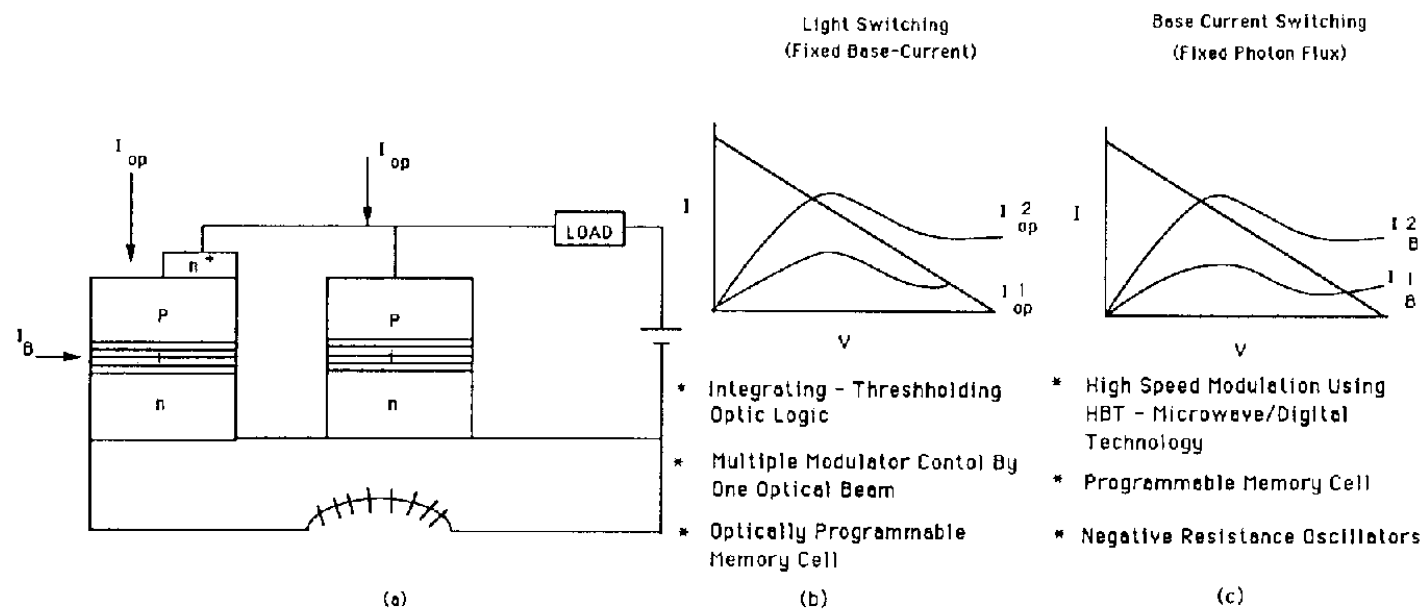

Figure 3 (a) MQW-HBT structure along with schematic of the switching operation by (b) optical power and (c) base current.

\section{THE MQW-HBT CONTROLLER-MODULATOR}

The potential of the negative resistance region of the I-V characteristics in a p-i-(MQW)-n structure was realized by Miller et al [4] and used to develop the self-electro-optic effect device (SEED). In order to make the SEED more compatible with the optical powers available in OEIC technology, it is important to have built-in electronic gain in the device. Gain is also essential for larger tolerance in the devices as well large fan-out and cascadability. Such gain can be realized by using an HBT with a MQW region in the base-collector region.

A schematic of the integrated MQW-HBT is shown in figure 3(a) along with the equivalent circuit. The MQW-HBT is shown in a circuit where it is controlling a modulator. It is clear that the controller and modulator can be realized by single-step epitaxy. The important point to realize is the presence of amplification of the photocurrent which allows low optical power switching. In a usual SEED the load resistances required are $\sim 100 \mathrm{~K} \Omega$ with an applied bias of $10-15$ volts. The load resistances are $\leq 5 \mathrm{~K} \Omega$ for the MQW-HBT's. An important asset of the MQW-HBT is that the I-V curves can be shifted either by optical power or through base current and as long as some optical power is present, the negative resistive region is maintained. In figure 3 we show schematically how the switching operation can be carried out using as input either the base current or the optical intensity. 


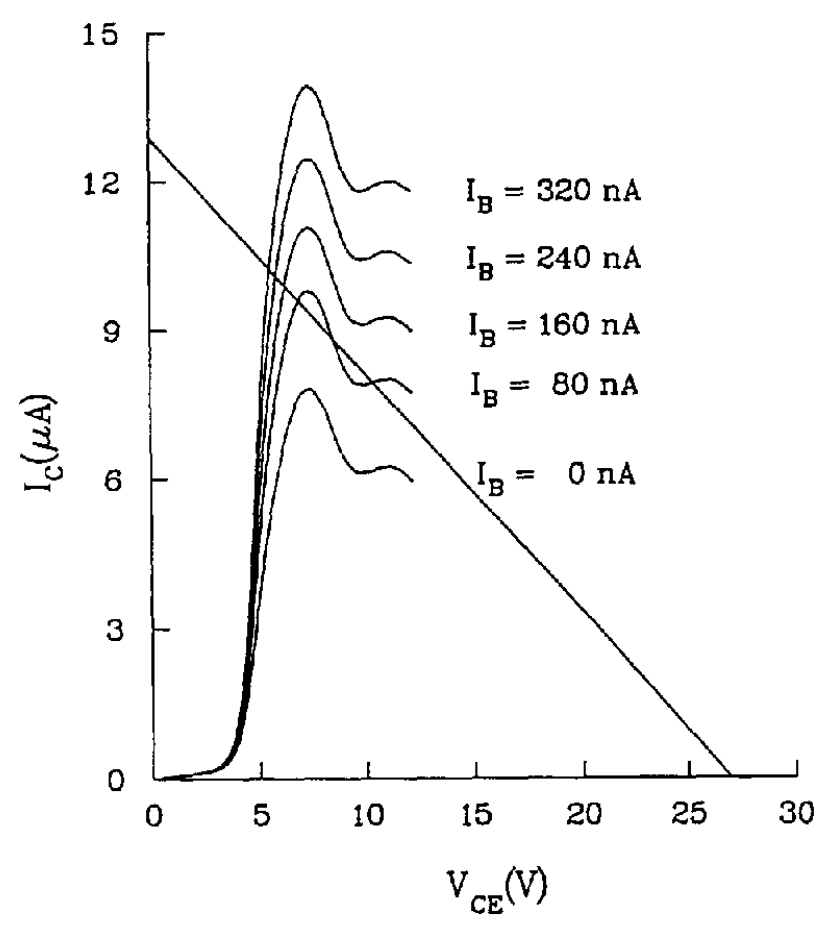

Figure 4 Measured I-V curves in a MQW-HBT with photoexcitation.

The heterostructure for the controller-modulator is grown by us using MBE. The collector region has the $0.6 \mu \mathrm{m}$ undoped $\mathrm{GaAs} / \mathrm{Al}_{0.3} \mathrm{Ga}_{0.7} \mathrm{As} \mathrm{MQW}$, which forms the essential element for the QCSE modulator and detector in the HBT. Typical current-voltage characteristics of a HBT device are measured in the dark. With photoexcitation a negative resistance region develops, as shown in figure 4. A maximum current gain of 60 was obtained at $I_{b}=17.5 \mu \mathrm{A}$ in a $50 \mu \mathrm{m}$ diameter device. A modulator device with $50 \mu \mathrm{m}$ diameter exhibits a very low leakage current (few pA at $8 \mathrm{~V}$ reverse bias). The large enhancement in the negative resistance region is quite obvious.

To measure the device operating characteristics, a tunable dye-laser and a spectrometer were used to select the optimal operating wavelength. It was found that at $\lambda=850 \mathrm{~nm}$ the photoresponse, both in the controller HBT and the modulator, exhibits a distinct NDR region. We briefly discuss some switching characteristics for ON/OFF optical switching. For our experiments the switching was performed between dark (OFF) and $10 / \mu \mathrm{W}$ (ON) optical light. We found the optimal bias conditions to be $R_{L}=15 \mathrm{~K} \Omega$ and $V_{\text {app }}=15 \mathrm{~V}$. An $8 \mathrm{~V}$ swing was obtained across the controller. This is large enough to change the transmittance across a $0.6 \mu \mathrm{m}$ thick MQW modulator by $50 \%$. It is evident that low power photonic switching is achieved due to the gain of the phototransistor controller.

The temporal response of the device was also studied. The response time is primarily limited by the RC time constant of the bipolar transistor which is estimated 


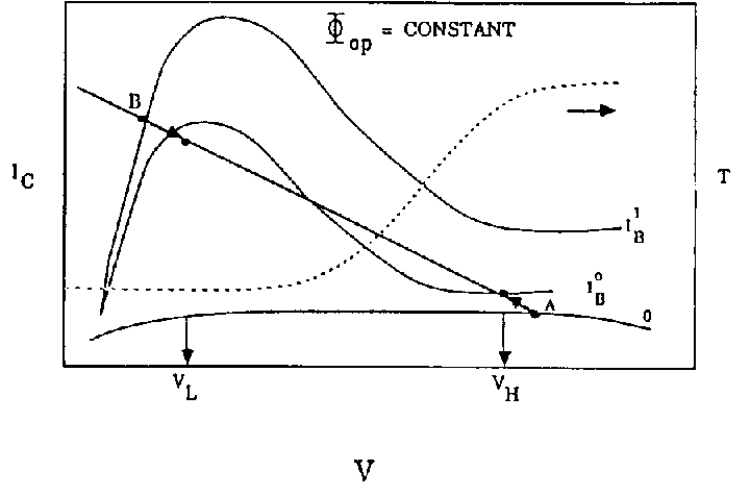

(a)

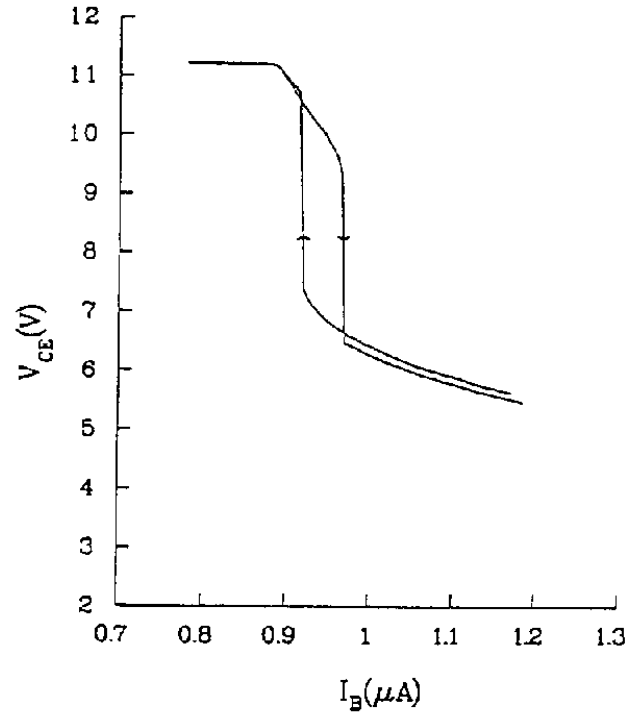

(b)

Figure 5 (a) I-V curves illustrating the operation of a flip-flop memory cell; (b) measured switching-holding and bistable characteristics.

to be $1 \mathrm{~ns}$ and the FWHM is $\sim 1.5 \mathrm{~ns}$. As more modulators are connected to one controller (lateral fanout), the response speed of the system becomes slower.

\section{OPTOELECTRONIC CONTROL AND AMPLIFICATION}

We expect that the large gain of the controller will allow a small change in the controller input intensity to produce a large change in the modulator output. Amplification values of 8 are obtained from these experiments. Such optical "amplification" can find important applications in optical systems. In addition, we have demonstrated that one controller can effectively control eight modulators. This is extremely important for parallel operations.

\section{PROGRAMMABLE MEMORY CELL}

When the input optical wavelength is below the $\mathrm{HH}$ exciton zero field energy, the I-V curves is such that a load line can have two stable points as shown in figure 5 (a). Conditions shown as input optical power of $\Phi_{o p}^{o}$ and base currents of $I_{B}^{o}$ are the holding conditions for the memory. To switch the memory to the high voltage value $V_{H}$, one can either switch off the hold light or lower the base current to zero. When the conditions are restored to $\Phi_{o p}^{o}$ and $I_{B}^{o}$, the device has the state $V_{H}$. For storing and holding the low voltage value $V_{L}$, either the base current or the optical power can 


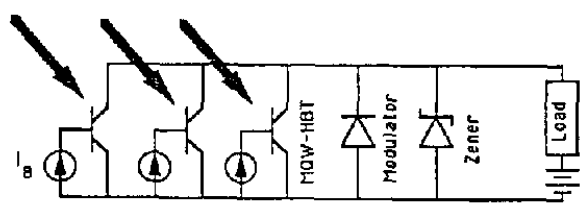

(a)

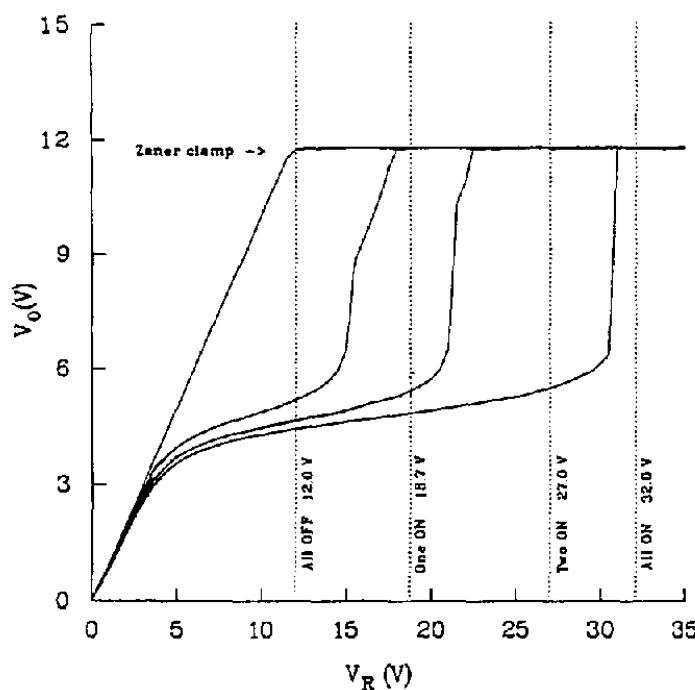

(b)

Figure 6 (a) Circuit diagram of a thresholding gate with three MQW-HBT devices and two p-i(MQW)-n modulators; (b) measured output characteristics of a thresholding gate. The horizontal axis represents the supply voltage.

be increased so that the only stable point for the load line is a low voltage value (B on the figure 5). Now when the hold conditions are restored the low voltage value is reached and held.

The MQW-HBT device discussed above is very well suited for the memory element because of the built in gain and consequent high noise margin [6]. It also has the practical aspect that the light level for all memory cells can be maintained at $I_{o p}^{0}$ and the memory values can be controlled through the base current. Also unlike the usual flip-flops, a single transistor is involved in the memory promising a high density level. In figure 5(b) we show our results for the switching and hold experiments.

\section{TUNABLE THRESHOLD LOGIC GATE}

Figure 6(a) shows the circuit diagram of an MQW-HBT threshold gate. The thick arrows in the diagram indicate optical input signals. For operation of the gate the wave length of the light should be above the excitonic peak, so that the complete heavy hole response appears in the photocurrent spectrum of the MQW-HBT. The current in the load resistor is a superposition of the currents in the MQW-HBTs. The p-i(MQW)-n diodes or modulators in parallel with the MQW-HBTs are used to modulate lights of the same wavelength. The photocurrent of the modulators also 
passes through the load, but the electronic gain of MQW-HBTs ensures that this photocurrent is much smaller than that of the MQW-HBT.

Figure 6(b) shows the experimental output characteristic for a 3 input threshold gate. A $100 \mathrm{~K} \Omega$ load resistance with a variable voltage supply was used. The zener diode connected in parallel with the MQW-HBTs prevents damage from accidental high voltages. A tunable $\mathrm{AlGaAs}$ laser a $\lambda \simeq 855 \mathrm{~nm}$ was used as the light source. When none of the MQW-HBTs are illuminated the output voltage increases linearly with the supply voltage, as there is no current in the circuit and hence no potential drop across the load.

From figure 6(b) it is seen that for a 12 volt supply the output is 1 when none of the inputs is 1 (illuminated), but becomes 0 when any of them is 1 . This is the NOR function. For a supply voltage of 18.7 , the circuit is an inverse carry gate as the output is 0 when two or more of the inputs are 1 , otherwise the output is 1 . When the supply voltage is about 27 the gate output is 0 whenever all of the inputs are 1 . Hence the gate now performs the NAND operation. Thus the function of the gate can be altered simply by changing the supply voltage. This property has some important application in electronic neural network implementation [7]. In addition, the access to the base terminal of the MQW-HBT allows fine tuning of the photocurrents in the MQW-HBTs, so that a small optical misalignment can be compensated by electrical means.

\section{ACKNOWLEDGMENTS}

This work was supported by the Air Force Office of Scientific Research under Contract AFOSR-88-0168.

\section{REFERENCES}

1. D.A.B. Miller, D. S. Chemla, T. C. Damen, A. C. Gossard, W. Wiegmann, T. H. Wood, and C. A. Burrus, Phys. Rev. Lett., 53, 2173 (1984).

2. D.A.B. Miller, D. S. Chemla, T. C. Damen, A. C. Gossard, W. Wiegmann, T. H. Wood, and C. A. Burrus, Phys. Rev. B, 32, 1093 (1985).

3. S. Hong and J. Singh, IEEE J. Quant. Elect., 25, 301 (1989).

4. D.A.B. Miller, D. S. Chemla, T. C. Damen, T. H. Wood, C. A. Burrus, A. C. Gossard, and W. Wiegmann, Opt. Lett., 9, 567 (1989).

5. S. Goswami, P. Bhattacharya and J. Singh, IEEE J. Quant. Elect., 27, 875 (1991).

6. W-Q. Li, S. Goswami, P. Bhattacharya and J. Singh, Electron. Lett., 27, 31 (1991).

7. J. Singh, S-C. Hong, P. K. Bhattacharya and R. Sahai, Appl. Optics, 17, 4554 (1988). 